\title{
Impact of Antibodies Against Polyethylene Glycol on the Pharmacokinetics of PEGylated Asparaginase in Children with Acute Lymphoblastic Leukaemia: A Population Pharmacokinetic Approach
}

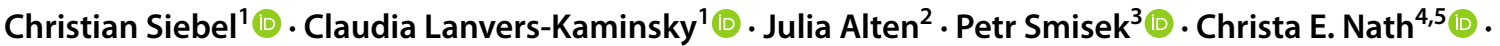

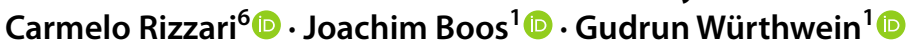

Accepted: 21 November 2021 / Published online: 8 December 2021

(c) The Author(s) 2021

\begin{abstract}
Background and Objectives Besides allergic reactions, antibodies against polyethylene glycol (PEG) have been associated with reduced PEG-asparaginase (PEG-ASNase) activity. Population pharmacokinetics (popPK) allow for an in-depth investigation of the influence of anti-PEG antibodies on PEG-ASNase pharmacokinetics.

Methods PEG-ASNase activity (6261 samples) and anti-PEG antibodies (2082/6412 samples prior to/post administration) in 1444 children with acute lymphoblastic leukaemia treated in the AIEOP-BFM ALL 2009 trial were evaluated. Patients received two doses of PEG-ASNase during induction $\left(2500 \mathrm{U} / \mathrm{m}^{2}\right.$, intravenous, biweekly) and a third dose during reinduction treatment. Anti-PEG IgG and IgM measured prior to and post administration were explored for their influence on the initial clearance of PEG-ASNase using a previously established popPK model. Categorical and continuous antibody data, including each isotype individually as well as in combination, were assessed.

Results High pre-existing levels of anti-PEG antibodies increase the initial drug clearance. Analysed separately, both anti-

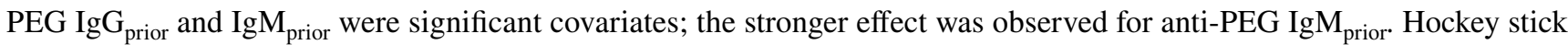

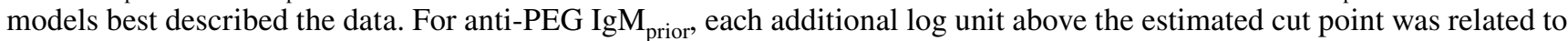
a 41.4\% increase in initial clearance after the first dose in induction. Antibody levels below the cut point were not associated with an effect on clearance. The combination of both isotypes did not provide additional information compared to anti-PEG $\operatorname{IgM}_{\text {prior }}$ alone. Antibody levels post administration were not associated with an effect on clearance.

Conclusion Pre-existing antibodies against PEG-ASNase significantly increased the initial clearance in a subgroup of patients showing high antibody levels. (Trial registration: EU clinical trials register; EudraCT No: 2007-004270-43; first registered 23 October 2009.)
\end{abstract}

\section{Introduction}

Polyethylene glycol (PEG)-conjugated asparaginase (PEGASNase, Oncaspar $\left.{ }^{\circledR}\right)$ is used in frontline treatment of children with acute lymphoblastic leukaemia (ALL) in a large number of contemporary treatment protocols [1-5]. PEGylation prolongs the circulation time of the enzyme and reduces its immunogenicity [6]. Though initially assumed to be biologically inert, PEG has been found to be immunogenic itself. Antibodies against PEG have been detected in patients upon treatment with PEG-ASNase, but also with other drugs

Gudrun Würthwein

wurthwg@uni-muenster.de

Extended author information available on the last page of the article

\section{Key Points}

High pre-existing levels of anti-PEG IgG and IgM antibodies significantly increase PEG-ASNase clearance during ALL induction treatment and thus affect the individually experienced treatment intensity.

This effect is especially pronounced for pre-existing anti-PEG IgM. In contrast, no effect was observed for antibodies identified in monitoring samples after PEGASNase administration.

It is recommended that pre-existing antibodies are taken into account when adjusting the PEG-ASNase dose to predefined activity ranges. 
such as PEGylated uricase or PEGylated recombinant phenylalanine ammonia lyase [7-11]. Furthermore, an increasing prevalence of antibodies against PEG has been observed in the general population (ranging from 0.2 to $72 \%$ ), which is attributed to the widespread use of PEG in cosmetics or food products (summarised in [12]).

In the AIEOP-BFM ALL 2009 trial, PEG-ASNase was applied in frontline therapy of children with ALL (EudraCT number: 2007-004270-43). As part of the trial, extensive monitoring of PEG-ASNase serum activity as well as antiPEG antibody levels was conducted. In a previous analysis of part of the study data performed by our working group, a high prevalence of anti-PEG $\operatorname{IgG}(13.9 \%)$ and $\operatorname{IgM}$ (29.1\%) prior to the first PEG-ASNase dose was observed [12]. Remarkably, these pre-existing anti-PEG IgG and IgM were associated with a reduction in PEG-ASNase activity following the first drug administration in induction treatment. One plausible explanation for the observed decrease in PEG-ASNase activity is accelerated elimination of antigen-antibody complexes.

Apart from pre-existing anti-PEG IgG and IgM, therapyinduced antibodies may also affect the distribution and elimination of PEG-ASNase. Hence, the aim of the present analysis was to provide a more detailed analysis of the potential influence of anti-PEG antibodies on PEG-ASNase pharmacokinetics in children with ALL. Specifically, the focus was on the role of anti-PEG antibodies in patients not affected by hypersensitivity reactions (clinically allergic reactions accompanied by a loss of ASNase activity) or clear silent inactivation (insufficient ASNase activity without allergy symptoms) [13]. Hypersensitivity reactions and silent inactivation constitute well-defined clinical categories associated with the development of distinct antibodies [7, 8, 14, 15]. However, even beyond these clear-cut phenomena, anti-PEG antibodies may have a quantifiable impact on the pharmacokinetics of PEG-ASNase and may in part explain the observed variability of PEG-ASNase pharmacokinetics in paediatric ALL patients [16].

Population pharmacokinetic (popPK) modelling was applied as a tool. A popPK model for PEG-ASNase during induction and reinduction treatment within the AIEOP-BFM ALL 2009 trial was recently published by our working group and served as a starting point to analyse the impact of antiPEG antibodies on the pharmacokinetics of PEG-ASNase $[16,17]$.

\section{Methods}

\subsection{Study Design}

Patients receiving induction and reinduction treatment according to the AIEOP-BFM ALL 2009 protocol (EudraCT number: 2007-004270-43) were included in the analysis. During induction treatment (protocol IA), patients received two doses of PEG-ASNase on days 12 and 26 (all patients). A third dose was given during reinduction treatment (protocol II) on day 8 (non-high-risk patients only). PEG-ASNase was applied as a $2 \mathrm{~h}$ infusion at a dose of $2500 \mathrm{U} / \mathrm{m}^{2} /$ day (max. $3750 \mathrm{U} /$ day). The analysis refers to samples from patients enrolled in the German and Czech part of the multinational trial. The trial was approved by the appropriate national and local review boards and conducted in accordance with the Declaration of Helsinki, the ICH Guideline for Good Clinical Practice and applicable national legislation. Informed consent was obtained from the parents or legal representatives of the children. As the analysis described here extended the original informed consent, an additional ethics committee vote was obtained (Ethics Committee of the Westfalen-Lippe Medical Association and the University of Münster; reference number 2021-071-f-S).

\subsection{Data}

Pharmacokinetic sampling was scheduled prior to the first PEG-ASNase administration in induction and reinduction treatment as well as 7 and 14 days after each PEG-ASNase administration. Samples were analysed for PEG-ASNase activity as well as anti-PEG IgG and anti-PEG IgM, as described in Section 1 of the Electronic Supplementary Material (ESM). Samples with PEG-ASNase activities below the lower limit of quantification (LLOQ) of the bioanalytical method were not included in the data set due to the small number of samples < LLOQ (3.1\% of all samples). Both anti-PEG IgG and IgM were analysed. Results of antibody measurements were expressed as the mean fluorescence intensity (MFI). Outliers were not defined for antiPEG antibody levels.

Criteria for the exclusion of patients and samples from the analysis are summarised in Fig. 1. As the analysis focused on the impact of anti-PEG antibodies on the standard pharmacokinetic processes of PEG-ASNase, samples obtained at or after the development of a hypersensitivity reaction or silent inactivation (defined as PEG-ASNase activity $<100 \mathrm{U} / \mathrm{L}$ within 8 days and/or PEG-ASNase activity < LLOQ within 15 days after administration) [13] were excluded.

Antibody levels obtained prior to the first PEG-ASNase administration per treatment phase and after each administration were included in the analysis as both continuous and categorical variables:

- $\log$ of the absolute value

- Log of the ratio of antibody levels after administration vs. prior to administration

- Categorised into $\leq$ cut point vs. $>$ cut point 
To categorise antibody levels, cut points previously defined for a PEG-naïve reference population were used (see [12] for details). Antibody levels were categorized as positive or negative for anti-PEG $\operatorname{IgG}(\mathrm{MFI}>8)$ or $\operatorname{IgM}$ $(\mathrm{MFI}>2)$.

\subsection{Pharmacokinetic Analysis}

\subsubsection{Population Pharmacokinetic Model}

The popPK model for PEG-ASNase previously published by our working group served as a starting point (described in detail in $[16,17])$. The model consists of a chain of 14 transit compartments to describe the increase in elimination over time (see Fig. S1 in the ESM). The initial clearance $\left(\mathrm{CL}_{\text {initial }}\right)$ and volume of distribution $(V)$ were found to be dependent on the administration (difference between the first and second doses in induction) and treatment phase (difference between induction and reinduction). Demographic covariates (age, sex) were also found to affect $\mathrm{CL}_{\text {initial }}$ [16].

\subsubsection{Anti-PEG Antibody Covariate Model Building}

Anti-PEG IgG and IgM measured prior to and after the administration of PEG-ASNase were evaluated as potential influential covariates affecting $\mathrm{CL}_{\text {initial. }}$. In a first step, the influences of anti-PEG $\operatorname{IgG}$ and $\operatorname{IgM}$ on $\mathrm{CL}_{\text {initial }}$ were tested separately with regard to the high collinearity between both potential covariates. In a second step, models that combine both antibody isotypes were evaluated. In both steps, antibodies were included in the models as either categorical or continuous (log-transformed) variables (see Section 2 in the ESM for details).

In the case of categorical covariates, distinct models for

- Anti-PEG IgG or IgM prior to PEG-ASNase administration ("anti-PEG IgG $\mathrm{Iprior}_{\text {"/ } / \text { anti-PEG IgM }}$ prior")

- Anti-PEG IgG or IgM after PEG-ASNase administration ("anti-PEG IgG after $_{\text {"/"anti-PEG IgM }}$ after")

were assessed.

Categorised antibody data were evaluated using the following proportional shift model:

$\mathrm{CL}_{\text {initial }}=\theta_{\text {pop }} \times\left(1+\theta_{\mathrm{AB}}\right)$

Here, $\theta_{\text {pop }}$ is the $\mathrm{CL}_{\text {initial }}$ value when antibody levels were below the respective cut point. In this case, $\theta_{\mathrm{AB}}$ is fixed at $0 . \theta_{\mathrm{AB}}$ is the fractional change in $\mathrm{CL}_{\text {initial }}$ when antibody levels were above the cut point.

In the case of continuous covariates, different models evaluating

- Anti-PEG $\operatorname{IgG}_{\text {prior }}$ or $\operatorname{IgM}_{\text {prior }}$
Fig. 1 Overview of the data set used to analyse the impact of anti-PEG antibodies on the pharmacokinetics of PEGASNase. ${ }^{(1)}$ Anti-PEG AB levels determined $>34$ days after administration were included in the data set (flagged with EVID = 2). ${ }^{(2)}$ Outliers identified during previous modelling steps (26 samples overall) [16]. $A B$ antibody, admin. administration, CWRES conditional weighted residual, $H S R$ hypersensitivity reaction, popPK population pharmacokinetic, SI silent inactivation

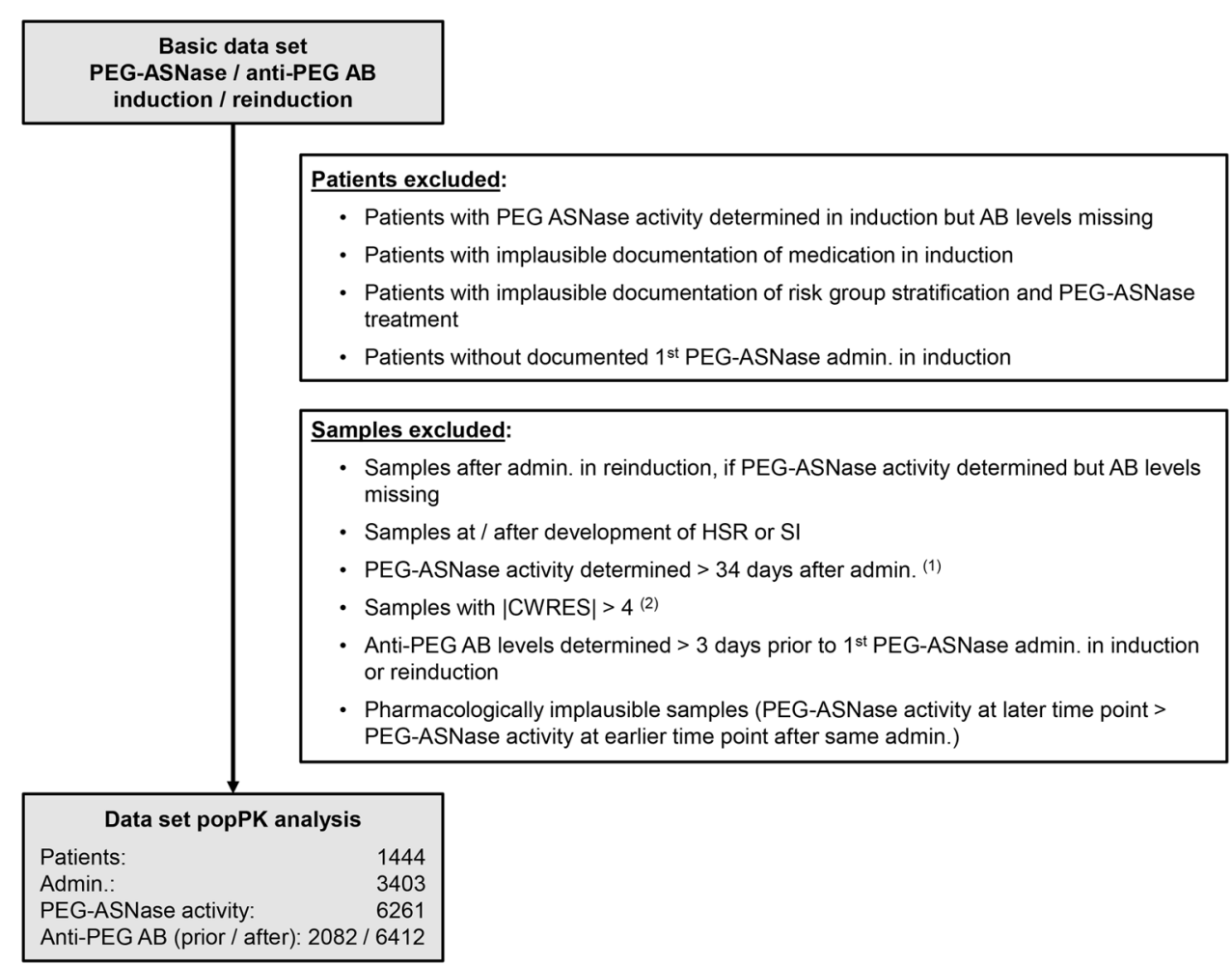


- Anti-PEG $\operatorname{IgG}_{\mathrm{after}}$ or $\operatorname{IgM}_{\mathrm{after}}$

- The ratio of antibody levels after administration vs. prior to administration

were assessed.

Antibody data were log-transformed due to the large range of antibody levels. Log-transformed variables were included in the model using linear covariate-parameter relationships, as described in

$\mathrm{CL}_{\text {initial }}=\theta_{\text {pop }} \times\left(1+\theta_{\mathrm{AB}} \times\left(\mathrm{AB}-\mathrm{AB}_{\text {median }}\right)\right)$

Here, $\theta_{\text {pop }}$ is the $\mathrm{CL}_{\text {initial }}$ value in individuals with antibody levels $(\mathrm{AB})$ equal to the median antibody level. $\theta_{\mathrm{AB}}$ is the fractional change in $\mathrm{CL}_{\text {initial }}$ with each $\log$ unit difference in the antibody level from the median antibody level in the population. When assessing the ratio of antibody levels after vs. prior to PEG-ASNase administration, centring of the effect of the covariate on the median was not performed. Thus, $\theta_{\text {pop }}$ reflected the $\mathrm{CL}_{\text {initial }}$ value when the antibody level after administration was not different from that prior to administration.

As the anti-PEG antibodies represent time-varying covariates, two extended approaches described by Wählby et al. [18] were additionally tested:

- In the first approach, the covariate effect was split into a baseline covariate effect of pre-existing antibodies $\left(\theta_{\mathrm{BCOV}}\right)$ and a change from baseline covariate effect expressed as the difference between the (log-transformed) antibodies after administration and the (log-transformed) antibodies prior to administration $\left(\theta_{\mathrm{DCOV}}\right)$; i.e. different relationships were estimated for between- and withinindividual variation in antibody levels:

$$
\begin{aligned}
\mathrm{CL}_{\text {initial }}= & \theta_{\text {pop }} \times\left(1+\theta_{\mathrm{BCOV}} \times\left(\mathrm{AB}_{\text {prior }}-\mathrm{AB}_{\text {prior median }}\right)\right. \\
& \left.+\theta_{\mathrm{DCOV}} \times\left(\mathrm{AB}_{\text {after }}-\mathrm{AB}_{\text {prior }}\right)\right)
\end{aligned}
$$

- In the second approach, an additional inter-individual variability (IIV) in the covariate effect $\left(\eta_{\mathrm{AB}, \mathrm{CLinitial}}\right)$ was included, i.e. the model allowed the magnitude of the antibody effect to differ between individuals with otherwise identical antibody levels. A possible interpretation of such a model could be the occurrence of antibodies with different affinities. This model required a logit transformation of the covariate-parameter relationship to ensure that individual $\mathrm{CL}_{\text {initial }}$ values remained positive (Eqs. 4, 5). As described above, centring of the covariate effect on the median was not performed when evaluating the change from baseline antibody levels.

Logit $=\theta_{\mathrm{AB}} \times\left(\mathrm{AB}-\mathrm{AB}_{\text {median }}\right)+\eta_{\mathrm{AB}, \text { CLinitial }}$

$$
\mathrm{CL}_{\text {initial }}=\theta_{\text {pop }} \times(0.5+\exp (\text { Logit }) /(1+\exp (\text { Logit })))
$$

\subsection{Model Selection Criteria}

Anti-PEG antibodies were tested for their significance in a univariate analysis. The best model from each modelling step was selected based on the likelihood ratio test $(\alpha=$ $0.01)$ (nested models), the Bayesian information criterion (BIC) (non-nested models), relative standard error estimates (\%RSE) of the model parameters, and graphical criteria (goodness-of-fit (GOF) plots). Once the final antibody covariate model was established, the demographic covariates age and sex were successively excluded from the model to test whether these covariate effects remained significant after the inclusion of the anti-PEG antibody effect. For key model development steps, prediction-corrected visual predictive checks (pcVPCs; $n=1000$ ) were performed. A bootstrap analysis $(n=1000)$ of the final antibody covariate model was conducted to calculate nonparametric confidence intervals for the model parameters.

\subsection{Impact of Anti-PEG Antibodies on PEG-ASNase Activity}

Monte Carlo simulations were performed to visualize the impact of anti-PEG antibodies on PEG-ASNase activity. Different antibody levels were selected for comparison. Other covariates were fixed to the median (BSA, age) or the most frequent value (sex). The standard dosing regimen of the AIEOP-BFM ALL 2009 trial was applied (2500 U/ $\mathrm{m}^{2} ; 2 \mathrm{~h}$ infusion). Further, a PEG-ASNase dose of $1500 \mathrm{U} /$ $\mathrm{m}^{2}$ ( $1 \mathrm{~h}$ infusion), as used in other treatment protocols, was also evaluated [4]. Overall, 1000 patients were simulated for each anti-PEG antibody level and dosing regimen.

\subsection{Software}

NONMEM (7.4.4, ICON, Dublin, Ireland), R (R-4.1.0, https://www.r-project.org/), R-Studio (1.4.1106, https:// www.rstudio.com/), PSN (4.9.0, https://uupharmacometrics. github.io/PsN/) and Pirana (3.0.0, https://www.certara.com/ software/pirana-modeling-workbench/) were used for popPK analysis and model diagnostics [19-23]. The first-order conditional estimation method with interaction $(\mathrm{FOCE}+\mathrm{I})$ was applied for model estimation. Data preparation was performed with SAS (9.4, TS1M4; SAS Institute GmbH, Heidelberg, Germany). Statistical and graphical analyses were performed with SAS and R. 


\section{Results}

\subsection{Data}

Overall, 1444 patients who received 3403 PEG-ASNase administrations during induction and reinduction treatment were included in the analysis (Table 1). 6261 measurements of PEG-ASNase activity were available from these patients (Table S1 in the ESM). Antibody levels were available for 2082 samples obtained prior to PEG-ASNase administration and 6412 samples obtained after administration, respectively. Table 2 gives an overview of the distribution of antiPEG IgG and IgM levels. The time course of antibody levels during induction and reinduction treatment is presented in Fig. 2.

\subsection{Population Pharmacokinetic Analysis}

\subsubsection{Anti-PEG Antibody Covariate Model Building}

- Modelling antibody isotypes separately

Table 1 Summary of patient characteristics

\begin{tabular}{ll}
\hline Variable & Number or median (range) \\
\hline Sex (male/female) & $842 / 602$ \\
Age (years) & $5.13(1.06-18.32)$ \\
Body surface area $\left(\mathrm{m}^{2}\right)$ & $0.78(0.41-2.58)$ \\
PEG-ASNase dose $(\mathrm{U})$ & $1950(720-5300)$ \\
PEG-ASNase dose $\left(\mathrm{U} / \mathrm{m}^{2}\right)$ & $2500(985-4464)$ \\
\hline
\end{tabular}

Different models for continuous and categorical anti-PEG IgG or IgM data were evaluated. For a detailed description of covariate model development, see Section 2 of the ESM. Overall, models including continuous antibody data were more informative than models including categorical data. Antibody levels obtained prior to PEG-ASNase administration were generally a better predictor of individual $\mathrm{CL}_{\text {initial }}$ compared to antibody levels obtained after drug administration or the ratio of antibody levels after vs. prior to administration. Modelling anti-PEG $\operatorname{IgG}_{\text {prior }}$ or $\operatorname{IgM}_{\text {prior }}$ together with an additional covariate effect for the difference between the antibody level after adminstration and the antibody level prior to administration (Eq. 3) did not lead to a better description of the variability in $\mathrm{CL}_{\text {initial }}$ compared to a model that only included prior antibody levels (anti-PEG $\operatorname{IgG}_{\text {prior: }}$ : $\Delta \mathrm{OFV}=-0.617$, degrees of freedom $(d f)=1$; anti-PEG $\left.\mathrm{IgM}_{\text {prior }}: \Delta \mathrm{OFV}=-0.450, d f=1\right)$. Likewise, adding an additional IIV in the covariate effect (Eqs. 4, 5) of anti-PEG $\mathrm{IgG}_{\text {prior }}$ or $\operatorname{IgM}_{\text {prior }}$, respectively, did not improve the model (increase in BIC > 8.8).

Next, the linear relationship between anti-PEG $\operatorname{IgG}_{\text {prior }}$ or $\operatorname{IgM}_{\text {prior }}$ and $\mathrm{CL}_{\text {initial }}$ was assessed for each of the three PEGASNase administrations separately. However, the covariate effect could only be estimated with sufficient precision following the first drug administration in induction, not following any of the other two PEG-ASNase administrations (here, the relative standard error of parameter estimates was $>50 \%$ ). These models were superior to models with the same covariate effect on all three administrations (anti-PEG $\mathrm{IgG}_{\text {prior }}: \Delta \mathrm{OFV}=-4.810, d f=0$; anti-PEG IgM $\operatorname{Irior}_{\text {pr }}: \Delta \mathrm{OFV}$ $=-6.248, d f=0)$.

Khalil et al. defined cut points to distinguish between antibody-positive and antibody-negative samples (cut point
Table 2 Overview of anti-PEG $\mathrm{IgG}$ and $\mathrm{IgM}$ antibodies

\begin{tabular}{llll}
\hline Parameter & Time point & $\begin{array}{l}\text { Induction } \\
\text { Number or median (range) }\end{array}$ \\
\hline Total number of samples & Prior to 1st admin. ${ }^{\text {a }}$ & 1444 & 638 \\
& After admin. & 5213 & 1199 \\
Number of samples per patient & & $5(1-8)$ & $3(1-5)$ \\
Anti-PEG IgG (MFI) & Prior to 1st admin. & $4.18(0.73-70.3)$ & $3.67(0.48-30.1)$ \\
& After 1st admin. & $3.30(0.29-51.6)$ & $2.94(0.36-30.7)$ \\
& After 2nd admin. & $3.12(0.39-110)$ & - \\
Anti-PEG IgM (MFI) & Prior to 1st admin. & $1.37(0.19-18.1)$ & $0.96(0.13-13.5)$ \\
& After 1st admin. & $0.83(0.06-22.9)$ & $0.68(0.07-8.28)$ \\
& After 2nd admin. & $0.76(0.07-18.0)$ & - \\
\hline
\end{tabular}

Admin. administration, ASNase asparaginase, $L L O Q$ lower limit of quantification, $M F I$ mean fluorescence activity, $P E G$ polyethylene glycol

${ }^{a}$ Samples taken prior to the second dose in induction were not available, as the measurement coincided with the day 14 sample after the first PEG-ASNase administration

${ }^{b}$ Data set includes 151 samples with information on antibody levels but without PEG-ASNase activity data or with PEG-ASNase activity < LLOQ 
Fig. 2 Changes in the anti-PEG $\operatorname{IgG}(\mathbf{a}, \mathbf{c})$ and $\operatorname{IgM}(\mathbf{b}, \mathbf{d})$ levels over time after the first dose. Upper panel: antibody levels during induction; lower panel: antibody levels during reinduction. Each blue line depicts the course of the antibody level over time after the first PEG-ASNase administration for an individual patient. The dashed grey line is a LOESS (smoothed) curve through the data. The nominal time point of the second dose in induction treatment is indicated as a dotted black line (14 days after the first administration). ASNase asparaginase, MFI mean fluorescence intensity, $P E G$ polyethylene glycol
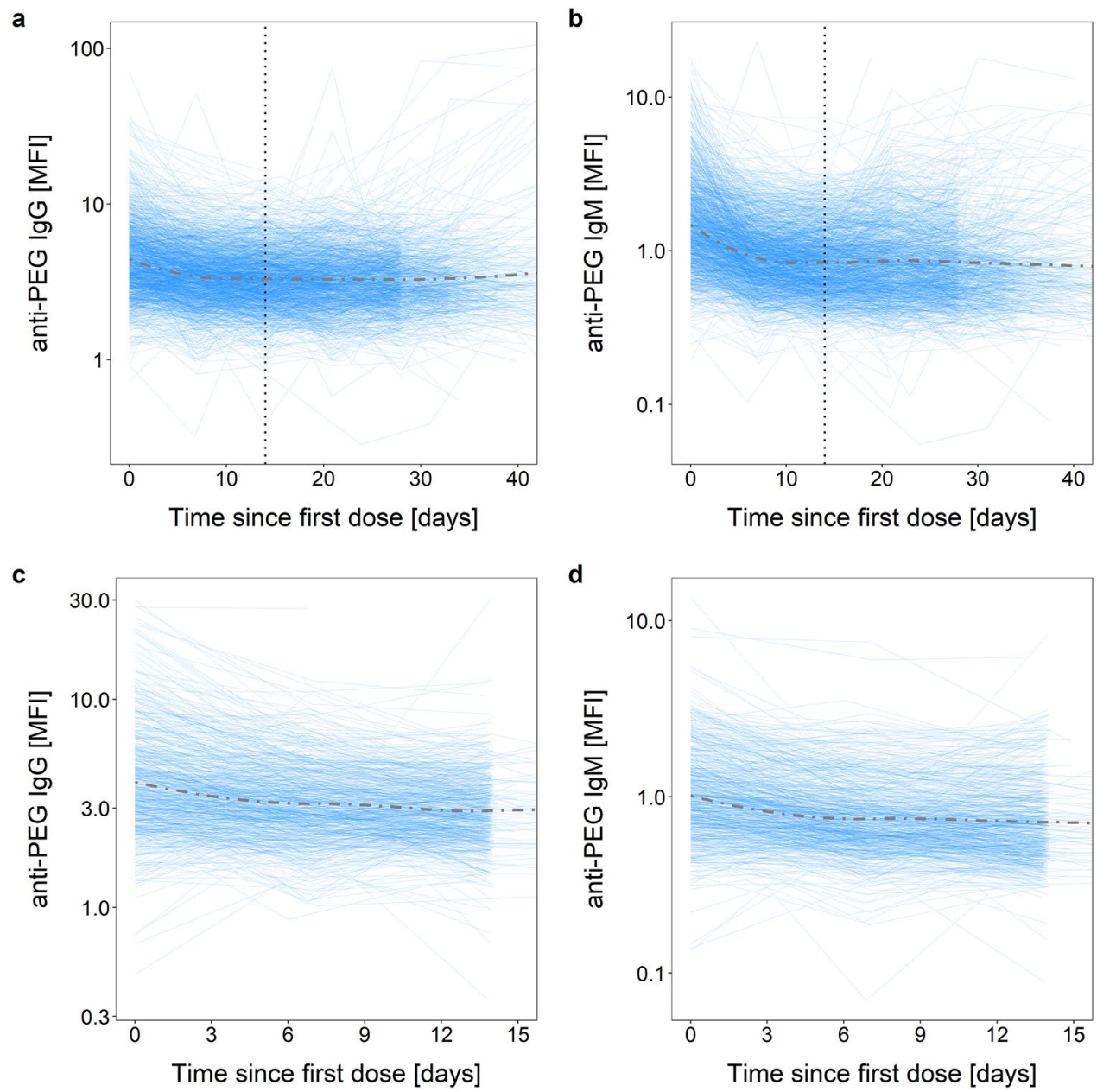

anti-PEG $\operatorname{IgG}_{\text {prior }}$ : $\mathrm{MFI}=8$; cut point anti-PEG $\operatorname{IgM}_{\text {prior }}$ : MFI = 2 (linear scale)) [12]. These cut points were taken into account by applying hockey stick models in place of the standard linear models. In these hockey stick models, antibody levels below the cut point showed no effect on PEG-ASNase pharmacokinetics, whereas (log-transformed) antibody levels above the cut point were linearly related to $\mathrm{CL}_{\text {initial }}$. In a first step, the cut points originally defined by Khalil et al. were employed; in a second step, these cut points were additionally re-estimated within NONMEM. For anti-PEG $\operatorname{IgG}_{\text {prior, }}$, a hockey stick model using the cut point defined by Khalil et al. was slightly superior to the standard linear model $(\Delta \mathrm{OFV}=-3.055, d f=0)$, and re-estimation of the cut point did not further improve the model. In contrast, taking into account the cut point defined by Khalil et al. for anti-PEG $\operatorname{IgM}_{\text {prior }}$ led to a more pronounced drop in the OFV ( $\Delta \mathrm{OFV}=-17.045, d f=0)$, and re-estimation of the cut point $(\mathrm{MFI}=3.67$ instead of 2 ; linear scale) within NONMEM improved the model even further $(\triangle \mathrm{OFV}=$ $-11.546, d f=1$ ). The hockey stick model including anti-

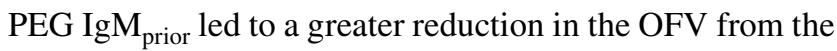

reference model than the model including anti-PEG $\operatorname{IgG}_{\text {prior }}$ $(\Delta \mathrm{OFV}=-49.184$ vs. $\Delta \mathrm{OFV}=-22.899)$.

\section{- Combination of both antibody isotypes}

Combination of the hockey stick models of both antibody isotypes did not provide additional information when compared to anti-PEG $\operatorname{IgM}_{\text {prior }}$ alone $(\Delta \mathrm{OFV}=-4.509, d f$ $=1$ ). Though both antibodies were informative for predicting individual $\mathrm{CL}_{\text {initial }}$, the results indicate that anti-PEG $\operatorname{IgM}_{\text {prior }}$ is a better predictor of $\mathrm{CL}_{\text {initial }}$.

Taken together, the hockey stick model with a linear relationship between (log-transformed) anti-PEG $\operatorname{IgM}_{\text {prior }}$ above the cut point of 3.67 (linear scale, expressed as MFI) and $\mathrm{CL}_{\text {initial }}$ was the best predictor of individual $\mathrm{CL}_{\text {initial }}$. On the basis of the data set, an effect of anti-PEG $\operatorname{IgM}_{\text {prior }}$ on $\mathrm{CL}_{\text {initial }}$ could only be estimated following the first dose in induction. Compared to the reference model (model without antibody covariates), inclusion of the covari-

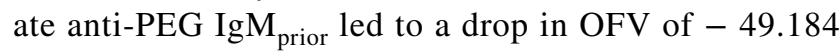
$(d f=2)$ (Table 3$)$. IIV in $\mathrm{CL}_{\text {initial }}$ marginally decreased 
from 25.8 to $25.4 \%$ and inter-occasion variability (IOV) decreased from 23.5 to $22.8 \%$.

\subsubsection{Model Evaluation}

Following the identification of the best anti-PEG antibody covariate model, an examination of whether the covariates age and sex remain significant after including the effect of anti-PEG IgM $\mathrm{M}_{\text {prior }}$ on $\mathrm{CL}_{\text {initial }}$ was performed. Elimination of the relationship between $\mathrm{CL}_{\text {initial }}$ and either age $(\Delta \mathrm{OFV}=$ $7.260, d f=1)$ or $\operatorname{sex}(\Delta \mathrm{OFV}=17.199, d f=1)$ significantly impaired the model.

The pcVPC demonstrates that the anti-PEG $\operatorname{IgM}_{\text {prior }}$ covariate model adequately predicts the data (Fig. 3). Just as for the published model (see [16]), day 14 samples after the second dose in induction were slightly underpredicted. GOF plots of the final anti-PEG $\operatorname{IgM}_{\text {prior }}$ covariate model are presented in the ESM (Fig. S2). The trend observable when plotting individual $\eta_{\text {CLinitial }}$ and $\kappa_{\text {CLinitial }}$ values against anti-

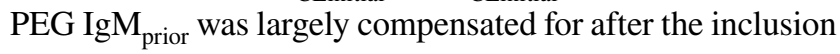
of the covariate effect, with the exception of the administration in reinduction (Fig. S3 in the ESM). Here, the remaining trend indicates an influence of high anti-PEG $\operatorname{IgM}_{\text {prior }}$ levels on $\mathrm{CL}_{\text {initial }}$, but this relationship was removed from the model due to the high parameter imprecision.

Table 3 summarises the results of the bootstrap analysis for the final covariate model. The median bootstrap estimates were in close agreement with the estimates for the final covariate model, indicating high model accuracy. Further, none of the $95 \%$ confidence intervals included the value
Table 3 Parameter estimates for the reference model and final covariate model, along with results of the bootstrap analysis

\begin{tabular}{|c|c|c|c|c|}
\hline \multirow[t]{2}{*}{ Parameter } & \multicolumn{2}{|c|}{ Typical values (\%RSE) } & \multicolumn{2}{|c|}{$\begin{array}{l}1000 \text { Bootstrap replicates ( } 97.8 \% \\
\text { successful) }\end{array}$} \\
\hline & Reference model & $\begin{array}{l}\text { Final covariate } \\
\text { model }\end{array}$ & Estimate & $95 \% \mathrm{CI}$ \\
\hline OFV & $73,391.518$ & $73,342.334$ & & \\
\hline$V\left(\mathrm{~L} / \mathrm{m}^{2}\right)$ & $1.71(1.4 \%)$ & $1.71(1.4 \%)$ & 1.71 & 1.65 to 1.78 \\
\hline $\mathrm{CL}_{\text {initial }}\left(\mathrm{L} / \mathrm{day} / \mathrm{m}^{2}\right)$ & $0.130(1.4 \%)$ & $0.128(1.4 \%)$ & 0.128 & 0.123 to 0.133 \\
\hline$Q_{\mathrm{tr}}\left(\mathrm{L} / \mathrm{day} / \mathrm{m}^{2}\right)$ & $0.965(2.5 \%)$ & $0.954(2.4 \%)$ & 0.957 & 0.901 to 1.01 \\
\hline$F_{\mathrm{BSA}}{ }^{\mathrm{a}}$ on $V$ & $1.60(2.1 \%)$ & $1.60(2.1 \%)$ & 1.60 & 1.54 to 1.67 \\
\hline$F_{\mathrm{BSA}}{ }^{\mathrm{a}}$ on $\mathrm{CL}_{\text {initial }}+Q_{\mathrm{tr}}$ & $1.50(2.1 \%)$ & $1.49(2.1 \%)$ & 1.49 & 1.43 to 1.55 \\
\hline Proportional error (\%) & $19.1(2.9 \%)$ & $0.191(2.8 \%)$ & 0.191 & 0.180 to 0.202 \\
\hline Additive error (U/L) & $5.37(47.3 \%)$ & $5.36(45.1 \%)$ & 5.16 & 2.23 to 11.5 \\
\hline$F\left(V_{\text {Induction 2.admin }}\right)^{\mathrm{b}}$ & $-0.155(6.8 \%)$ & $-0.158(6.7 \%)$ & -0.157 & -0.179 to -0.136 \\
\hline$F\left(V_{\text {Reinduction }}\right)^{\mathrm{b}}$ & $-0.284(3.8 \%)$ & $-0.288(3.7 \%)$ & -0.287 & -0.306 to -0.265 \\
\hline$F\left(\mathrm{CL}_{\text {Initial induction 2.admin }}\right)^{\mathrm{b}}$ & $-0.125(10.6 \%)$ & $-0.107(12.8 \%)$ & -0.108 & -0.135 to -0.078 \\
\hline$F\left(\mathrm{CL}_{\text {Initial reinduction }}\right)^{\mathrm{b}}$ & $-0.451(3.3 \%)$ & $-0.438(3.4 \%)$ & -0.439 & -0.469 to -0.407 \\
\hline$F(\text { age }>8 \text { years })^{\mathrm{c}}$ & $0.010(42.7 \%)$ & $0.011(38.8 \%)$ & 0.011 & 0.003 to 0.021 \\
\hline$F(\operatorname{sex})^{\mathrm{d}}$ & $-0.069(24.1 \%)$ & $-0.070(23.4 \%)$ & -0.070 & -0.101 to -0.036 \\
\hline$F\left(\operatorname{IgM}_{\text {Prior induction 1.admin }}>\mathrm{CP}\right)^{\mathrm{e}}$ & - & $0.414(18.0 \%)$ & 0.416 & 0.237 to 0.831 \\
\hline $\mathrm{CP}^{\mathrm{f}}$ & - & $1.30(2.5 \%)$ & 1.30 & 0.984 to 1.68 \\
\hline IIV CL $\mathrm{CL}_{\text {initial }}(\%)$ & $25.8(4.5 \%)$ & $25.4(4.5 \%)$ & 25.4 & 22.9 to 27.7 \\
\hline IOV $V(\%)$ & $11.9(9.7 \%)$ & $11.9(9.6 \%)$ & 11.8 & 9.6 to 14.5 \\
\hline IOV CL $\mathrm{L}_{\text {initial }}(\%)$ & $23.5(5.1 \%)$ & $22.8(4.9 \%)$ & 22.9 & 20.7 to 25.2 \\
\hline
\end{tabular}

Typical values for $V, \mathrm{CL}_{\text {initial }}$ and $Q_{\mathrm{tr}}$ are reported for a child with $\mathrm{BSA}=1 \mathrm{~m}^{2}$ for better comparison admin administration, ASNase asparaginase, BSA body surface area, 95\% CI 95\% confidence interval, $C L_{\text {initial }}$ initial clearance of PEG-ASNase, $C P$ cut point, $I g M_{\text {prior }}$ anti-PEG IgM level prior to first PEGASNase administration in induction or reinduction, $I I V$ inter-individual variability, $I O V$ inter-occasion variability, $O F V$ objective function value, $P E G$ polyethylene glycol, $Q_{t r}$ intercompartmental clearance, $\% R S E$ percent relative standard error, $V$ volume of distribution

${ }^{a}$ Linear increase in $V / \mathrm{CL}_{\text {initial }} / Q_{\text {tr }}$ with BSA (centred on the median)

${ }^{\mathrm{b}}$ Fractional change in $V / \mathrm{CL}_{\text {initial }}$ compared to the first administration in induction

${ }^{c}$ Linear increase in $\mathrm{CL}_{\text {initial }}$ for patients older than 8 years

${ }^{\mathrm{d}}$ Fractional change in $\mathrm{CL}_{\text {initial }}$ for females

${ }^{\mathrm{e}}$ Linear increase in $\mathrm{CL}_{\text {initial }}$ per $\log$ unit of anti-PEG IgM $\mathrm{M}_{\text {prior }}$ above the cut point following the first administration in induction

${ }^{\mathrm{f}}$ Cut point on $\log$ scale 
Fig. 3 Prediction-corrected visual predictive check for the final anti-PEG IgM prior $_{\text {}}$ covariate model stratified by PEG-ASNase administration. Red line is the median of the observations; blue lines are the 2.5 and 97.5 percentiles of the observations; shaded areas are the $95 \%$ confidence intervals for simulated data (1000 simulated datasets) for the corresponding percentiles; dots are observed PEG-ASNase activity levels. ASNase asparaginase, $P E G$ polyethylene glycol

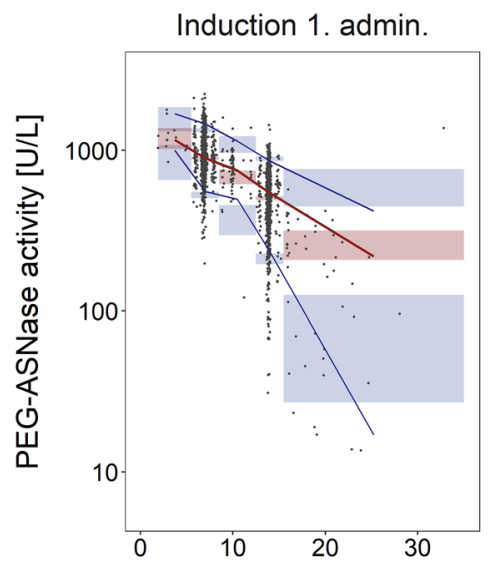

Induction 2. admin.

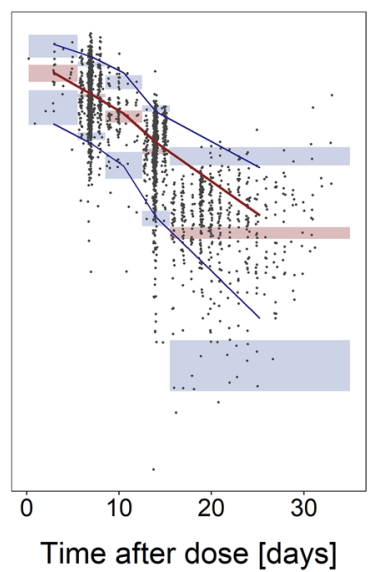

Reinduction

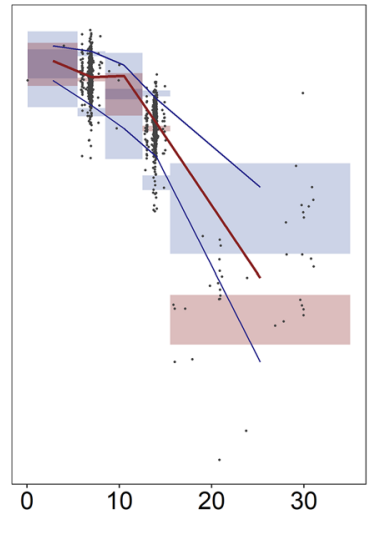

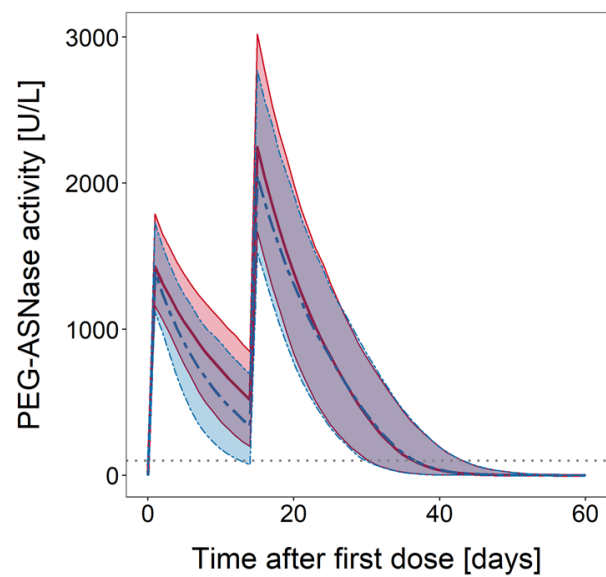

Fig. 4 Simulated PEG-ASNase activity over time in induction treatment. Dosing regimens applying PEG-ASNase at $2500 \mathrm{U} / \mathrm{m}^{2}$ over $2 \mathrm{~h}$ (a) and $1500 \mathrm{U} / \mathrm{m}^{2}$ over $1 \mathrm{~h} \mathrm{(b)}$ were evaluated. Patients were simu-

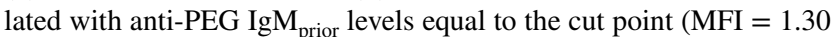
on the log scale; 3.67 on the linear scale) or one log unit above the cut point (MFI $=2.30$ on the log scale; 9.97 on the linear scale). For

zero, thus proving the significance of the covariate effects in the model.

\subsection{Impact of Anti-PEG Antibodies}

According to the final covariate model, each additional

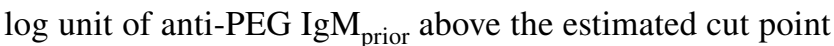
(i.e. an increase in anti-PEG $\operatorname{IgM}_{\text {prior }}$ from 1.30 to 2.30 on the log scale or, equivalently, from 3.67 to 9.97 on the linear scale) was related to an increase in $\mathrm{CL}_{\text {initial }}$ of $41.4 \%$. Figure 4 presents the PEG-ASNase activity simulated for hypothetical patients with anti-PEG $\operatorname{IgM}_{\text {prior }}$ levels equal to the cut point or one $\log$ unit above the cut point. For a dose of $2500 \mathrm{U} / \mathrm{m}^{2}$ median simulated PEG-ASNase, trough levels at day 14 after the first administration in induction b

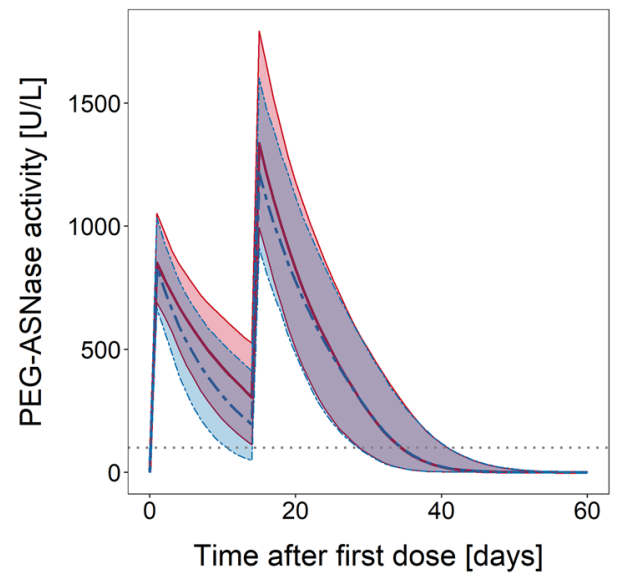

each antibody level and dosing regimen, 1000 patients were simulated. The solid red lines and dashed blue lines indicate the median PEG-ASNase activity at each antibody level. The shaded red and blue areas depict the respective $95 \%$ prediction intervals. The dotted grey lines indicate the threshold PEG-ASNase activity of 100 U/L. ASNase asparaginase, PEG polyethylene glycol

were $517 \mathrm{U} / \mathrm{L}$ (interquartile range (IQR) 391-632 U/L, anti-

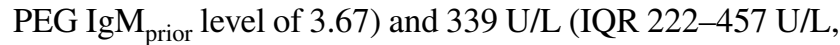

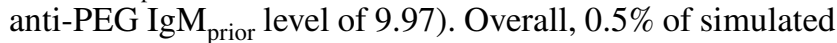
patients with an anti-PEG IgM $\mathrm{M}_{\text {prior }}$ level of 3.67 fell below the threshold of $100 \mathrm{U} / \mathrm{L}$, which is considered to be required for complete asparagine depletion [13], compared to $4.9 \%$ of

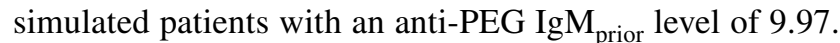
For a dose of $1500 \mathrm{U} / \mathrm{m}^{2}$ median PEG-ASNase, trough levels 14 days after the first administration were $305 \mathrm{U} / \mathrm{L}$ (IQR 241-367 U/L) and 194 U/L (IQR 135-262 U/L); 1.7\% and $14.9 \%$ of the patients were below $100 \mathrm{U} / \mathrm{m}^{2}$.

Simulations are consistent with data observed in the AIEOP-BFM ALL 2009 trial: overall, $0.86 \%$ of patients

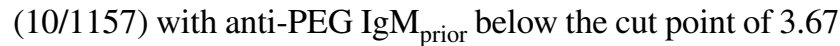
and $3.85 \%$ of patients (5/130) with anti-PEG IgM $\operatorname{srior}_{\text {above }}$ 
the cut point of 3.67 had PEG-ASNase activities $<100 \mathrm{U} / \mathrm{L}$ at day 13-15 after the first administration in induction (odds ratio $=4.59 ; 95 \%$ confidence interval: $1.54-13.6$; see Table S5 in the ESM).

\section{Discussion}

Paediatric ALL patients considerably benefit from intensive PEG-ASNase therapy associated with prolonged asparagine depletion [24-27]. Maintenance of adequate PEG-ASNase treatment intensity is therefore essential and requires knowledge of factors that interfere with PEG-ASNase pharmacokinetics, which may necessitate adjustment of dosages. On the other hand, individualisation of PEG-ASNase treatment has been proposed in order to reduce PEG-ASNase doses and such treatment-related toxicities, but also to decrease the costs of PEG-ASNase therapy [4, 28]. Adjustment of individual PEG-ASNase doses on the basis of therapeutic drug monitoring has recently been clinically evaluated within the Dutch Childhood Oncology Group ALL-11 protocol [4]. Moreover, Kloos et al. developed dosing guidelines on the basis of a popPK model [29]. Obviously, such dosing guidelines need to take into account all factors that significantly affect PEG-ASNase pharmacokinetics to reduce the risk of underdosing.

Anti-PEG antibodies have been associated with the development of clinically overt hypersensitivity reactions against PEG-ASNase as well as with a lack of enzyme activity (socalled silent inactivation) [7, 8]. Apart from hypersensitivity reactions and silent inactivation, the observation of reduced PEG-ASNase activities in patients with pre-existing antiPEG antibodies indicates that antibodies against the PEG moiety also exert a more general effect on the pharmacokinetics [12]. Using popPK modelling, we were able to investigate in detail the relationship between anti-PEG antibodies and PEG-ASNase elimination and to quantify their impact on individual treatment intensity.

Different functional forms for both antibody levels obtained prior to as well as after PEG-ASNase administration were explored to characterise their impact on $\mathrm{CL}_{\text {initial }}$. Consistently, for both the IgG and the IgM isotypes, the strongest relationship was between $\mathrm{CL}_{\text {initial }}$ and the antibody levels obtained prior to drug administration when considered as continuous data. However, on the basis of the data, the relationship between $\mathrm{CL}_{\text {initial }}$ and anti-PEG $\operatorname{IgG}_{\text {prior }}$ or $\operatorname{IgM}$ prior could only be precisely estimated following the first dose in induction, not following any of the other two drug administrations. With regard to the second dose in induction, the missing predictive power of antibody levels prior to first dosing is plausible, as the time lag between antibody determination ( $\leq 3$ days prior to the first dose) and second dosing was about 2 weeks, and the antibody concentration can vary substantially during this time as a consequence of the administration of the first PEG-ASNase dose. However, regarding the administration in reinduction, one could expect that anti-PEG $\operatorname{IgG}_{\text {prior }}$ or $\operatorname{IgM}_{\text {prior }}$ (determined $\leq 3$ days prior to dosing in reinduction) exert a similar influence on $\mathrm{CL}_{\text {initial }}$ to their effects following the first dose in induction (median 150 days apart). Presumably, the small number of samples with high antibody levels in reinduction impedes precise estimation of the covariate-parameter relationship. Thus, only 89 samples prior to administration in reinduction showed anti-PEG IgM levels above the cut point defined by Khalil et al., compared to 434 samples prior to the first dose in induction [12]. Nevertheless, it has to be taken into account that the treatment context (comedication, disease stage, physical condition) strongly differs between treatment phases, which might influence the relationship between antiPEG antibodies and $\mathrm{CL}_{\text {initial }}$ as well as the antibody level itself. Of note, an association between the treatment context and PEG-ASNase activity has also been observed, with increasing PEG-ASNase activity seen in reinduction compared to the PEG-ASNase activity seen following the first dose in induction [16, 29].

Though the relationship between $\mathrm{CL}_{\text {initial }}$ and anti-PEG antibodies exists for both isotypes, anti-PEG $\operatorname{IgM}_{\text {prior }}$ was a better predictor of individual $\mathrm{CL}_{\text {initial }}$. When both antibodies were combined, anti-PEG $\operatorname{IgG}_{\text {prior }}$ did not add any further information compared to anti-PEG IgM prior $_{\text {alone (see Sec- }}$ tion 2 in the ESM). According to the final covariate model, anti-PEG $\operatorname{IgM}_{\text {prior }}$ levels below the cut point of 3.67 do not exert an influence on $\mathrm{CL}_{\text {initial }}$, whereas $\mathrm{CL}_{\text {initial }}$ increases for

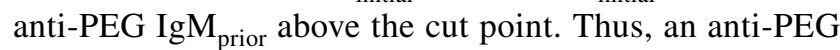
$\mathrm{IgM}_{\text {prior }}$ level of 9.97 (an increase of one log unit) is associated with an increase in $\mathrm{CL}_{\text {initial }}$ of $41.4 \%$ compared to the $\mathrm{CL}_{\text {initial }}$ associated with an antibody level of 3.67 or below. Kloos et al. explored the influence of antibodies against PEG-ASNase on drug CL during popPK model building [29]. In contrast to our findings, the relationship between antibodies and CL turned out to be nonsignificant. However, in their study, antibodies were only determined post dosing, and pre-existing antibodies were not assessed. Furthermore, the exact moiety recognised by the antibodies (i.e. PEG or the asparaginase itself) has not been characterised, precluding any comparison between the two studies. Poppenborg and colleagues explored the impact of anti-PEG IgM on PEG-ASNase activity in mice pre-sensitized with $40 \mathrm{kD}$ PEG-diol [30]. An accelerated decrease in PEG-ASNase activity in sera from successfully sensitised mice could not be observed, indicating that pre-existing anti-PEG IgM did not affect the pharmacokinetics of PEG-ASNase. Whether the experimental setup is appropriate to reflect the situation in humans is, however, uncertain. Pre-sensitisation with PEG-diol did not induce a constant response to PEG, and the amount of induced anti-PEG IgM might have been too low 
to affect PEG-ASNase activity. As suggested by our model, antibody levels need to exceed a certain threshold to exert an influence on $\mathrm{CL}_{\text {initial. }}$.

During the modelling process, pre-existing antibody levels as well as the entire time course of antibody levels subsequent to PEG-ASNase administration were evaluated as covariates. Neither anti-PEG IgG nor IgM as a time-varying covariate post dosing were predictive for $\mathrm{CL}_{\text {initial. }}$. For the example of infliximab, Edlund et al. have shown that the total anti-drug antibody concentration was a more appropriate descriptor of infliximab CL, whereas measurement of the unbound antibody concentration underestimated the effect on CL [31]. A similar explanation might apply here, as only the free antibody fraction was determined by the analytical assay, not the fraction of antibodies bound to PEG-ASNase. Moreover, an induction of antibody levels following PEGASNase administration might not be detectable by measurement of the unbound antibody level, thus masking the true relationship between antibodies and $\mathrm{CL}_{\text {initial }}$.

The pharmacokinetics of PEG-ASNase have been found to be dependent on the administration and treatment phase $[16,29,32]$. Various factors such as differences in the comedication have been discussed as possible underlying causes of the variations in PEG-ASNase pharmacokinetics. On the basis of our analysis, anti-PEG antibodies explain only a small part of the observed alterations in PEG-ASNase pharmacokinetics. Thus, inclusion of anti-

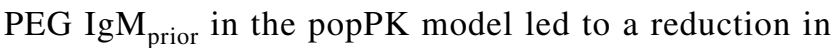
the factor describing the change in $\mathrm{CL}_{\text {initial }}$ between the first and second PEG-ASNase administrations in induction from -0.125 to -0.107 .

It should be emphasized that the increase in $\mathrm{CL}_{\text {initial }}$ associated with anti-PEG antibodies only affects a minority of patients who show very high pre-existing antibody levels. For the data set used for popPK analysis, only 146/1444 patients in induction and 9/638 patients in reinduction showed anti-PEG IgM $\mathrm{Mrior}_{\text {p }}$ levels above the finally estimated cut point. This is reflected in the very small decrease in the random variability of $\mathrm{CL}_{\text {initial }}$ following the inclusion of the covariate effect in the popPK model. Compared to the reference model, the inclusion of the effect of anti-PEG IgM $\operatorname{Igrior}_{\text {}}$ on $\mathrm{CL}_{\text {initial }}$ led to a decrease in IIV from 25.8 to $25.4 \%$ and a reduction in IOV from 23.5 to $22.8 \%$ (Table 3). Though the absolute number of affected patients is rather small, our analysis nevertheless indicates that these patients show an increased risk of inadequate PEG-ASNase therapy (Fig. 4). In particular, application of low PEG-ASNase doses such as $1500 \mathrm{U} / \mathrm{m}^{2}$ harbour the risk that an increased proportion of patients will exhibit insufficient PEG-ASNase activity, again highlighting the value of PEG-ASNase therapeutic drug monitoring. Adjustment of the very first PEG-ASNase dose on the basis of pre-existing anti-PEG antibody levels might be an additional option to further optimise PEG-ASNase therapy, but this is hampered by the lack of widespread assays for anti-PEG antibody measurement.

\section{Conclusion}

In conclusion, popPK modelling showed that pre-existing anti-PEG IgG and IgM both influence the pharmacokinetics of PEG-ASNase, with the IgM isotype being the better predictor for individual $\mathrm{CL}_{\text {initial }}$ values. Treatment intensity is considerably affected in a subgroup of patients that show very high levels of pre-existing antibodies. In these patients, the final covariate model allows the activity-time course of PEG-ASNase to be more precisely predicted and highlights the value of monitoring anti-PEG antibody levels, at least prior to the very first PEG-ASNase administration.

Supplementary Information The online version contains supplementary material available at https://doi.org/10.1007/s13318-021-00741-w.

Acknowledgements The authors would like to thank Natalia Günter, Stephanie Hoogestraat, Petra Schulze Westhoff, Andrea Rüffer and Sandra Schulz for excellent technical assistance, and Jana Golitsch and Sebastian Meschke for documentation and data management. The authors also express thanks to the patients and their families participating in the AIEOP BFM ALL 2009 trial and the physicians and nurses of all hospitals for their contribution.

Funding Open Access funding enabled and organized by Projekt DEAL.

\section{Declarations}

Funding Assay development and monitoring of anti-PEG antibodies within the AIEOP-BFM ALL 2009 trial was supported by Deutsche José Carreras Leukämie-Stiftung e.V. (DJCLSR 13/01). Additional funding was provided by Servier Deutschland $\mathrm{GmbH}$ to perform the population pharmacokinetic analysis described here. Christa E. Nath is supported by the Sydney Children's Hospital Network Foundation. Asparaginase monitoring in Australia was supported by funding from the Kids Cancer Alliance, a translational cancer research centre of the Cancer Institute of NSW.

Conflicts of interest CLK held invited talks for Sigma Tau, Erytech, Jazz Pharmaceuticals and Servier and received honoraria for consultancy from Erytech. CR received honoraria for consultancy and fees for invited lectures from Jazz Pharmaceuticals and Servier. JB serves as a consultant for Servier, held invited lectures and received a research grant-always in the form of an institutional grant in the context of ASNase drug monitoring. CS, JA, PS, CN and GW declare no conflicts of interest.

Ethics approval The AIEOP-BFM ALL 2009 trial (EudraCT No: 2007004270-43) was approved by each national and local review board and was conducted in accordance with the Declaration of Helsinki and applicable national legislation. Informed consent was obtained from the parents or guardians of each patient included in the study, as required by ethical standards and national guidelines. For the pharmacokinetic analysis described here, an additional vote of the Ethics Committee of 
the Westfalen-Lippe Medical Association and the University of Münster was obtained (reference number 2021-071-f-S).

Consent to participate Informed consent was obtained from the parents or legal representative of each patient included in the study, as required by ethical standards and national guidelines.

\section{Consent for publication Not applicable.}

Availability of data and material The datasets generated and/or analysed during the current study are not publicly available due to data protection rules and the fact that the complexity of the data does not allow for full anonymization.

Code availability The NONMEM code for the final anti-PEG antibody covariate model is provided in the ESM.

Author's contribution JB, CLK, CS and GW designed the research. JA and PS collected the data. CS and GW performed the pharmacokinetic analysis. Results were discussed and interpreted by all authors. CS and GW wrote the draft manuscript; all authors reviewed the manuscript and approved the final version of the manuscript.

Open Access This article is licensed under a Creative Commons Attribution-NonCommercial 4.0 International License, which permits any non-commercial use, sharing, adaptation, distribution and reproduction in any medium or format, as long as you give appropriate credit to the original author(s) and the source, provide a link to the Creative Commons licence, and indicate if changes were made. The images or other third party material in this article are included in the article's Creative Commons licence, unless indicated otherwise in a credit line to the material. If material is not included in the article's Creative Commons licence and your intended use is not permitted by statutory regulation or exceeds the permitted use, you will need to obtain permission directly from the copyright holder. To view a copy of this licence, visit http://creativecommons.org/licenses/by-nc/4.0/.

\section{References}

1. Place AE, Stevenson KE, Vrooman LM, et al. Intravenous pegylated asparaginase versus intramuscular native Escherichia coli L-asparaginase in newly diagnosed childhood acute lymphoblastic leukaemia (DFCI 05-001): a randomised, open-label phase 3 trial. Lancet Oncol. 2015;16(16):1677-90. https://doi.org/10. 1016/S1470-2045(15)00363-0.

2. Tram Henriksen L, Gottschalk Højfeldt S, Schmiegelow K, et al. Prolonged first-line PEG-asparaginase treatment in pediatric acute lymphoblastic leukemia in the NOPHO ALL2008 protocol-pharmacokinetics and antibody formation. Pediatr Blood Cancer. 2017. https://doi.org/10.1002/pbc.26686.

3. Rizzari C, Lanvers-Kaminsky C, Valsecchi MG, et al. Asparagine levels in the cerebrospinal fluid of children with acute lymphoblastic leukemia treated with pegylated-asparaginase in the induction phase of the AIEOP-BFM ALL 2009 study. Haematologica. 2019;104(9):1812-21. https://doi.org/10.3324/ haematol.2018.206433.

4. Kloos RQH, Pieters R, Jumelet FMV, de Groot-Kruseman HA, van den Bos C, van der Sluis IM. Individualized asparaginase dosing in childhood acute lymphoblastic leukemia. J Clin Oncol. 2020;38(7):715-24. https://doi.org/10.1200/JCO.19. 02292 .
5. Maloney KW, Devidas M, Wang C, et al. Outcome in children with standard-risk B-cell acute lymphoblastic leukemia: results of children's oncology group trial AALL0331. J Clin Oncol. 2020;38(6):602-12. https://doi.org/10.1200/JCO.19.01086.

6. Harris JM, Chess RB. Effect of pegylation on pharmaceuticals. Nat Rev Drug Discov. 2003;2(3):214-21. https://doi.org/10.1038/ nrd1033.

7. Armstrong JK, Hempel G, Koling S, et al. Antibody against poly(ethylene glycol) adversely affects PEG-asparaginase therapy in acute lymphoblastic leukemia patients. Cancer. 2007;110(1):103-11. https://doi.org/10.1002/cncr.22739.

8. Liu Y, Smith CA, Panetta JC, et al. Antibodies predict pegaspargase allergic reactions and failure of rechallenge. J Clin Oncol. 2019;37(23):2051-61. https://doi.org/10.1200/JCO.18.02439.

9. Ganson NJ, Kelly SJ, Scarlett E, Sundy JS, Hershfield MS. Control of hyperuricemia in subjects with refractory gout, and induction of antibody against poly(ethylene glycol) (PEG), in a phase I trial of subcutaneous PEGylated urate oxidase. Arthritis Res Ther. 2006;8(1):R12. https://doi.org/10.1186/ar1861.

10. Hershfield MS, Ganson NJ, Kelly SJ, Scarlett EL, Jaggers DA, Sundy JS. Induced and pre-existing anti-polyethylene glycol antibody in a trial of every 3-week dosing of pegloticase for refractory gout, including in organ transplant recipients. Arthritis Res Ther. 2014;16(2):R63. https://doi.org/10.1186/ar4500.

11. Longo N, Harding CO, Burton BK, et al. Single-dose, subcutaneous recombinant phenylalanine ammonia lyase conjugated with polyethylene glycol in adult patients with phenylketonuria: an open-label, multicentre, phase 1 dose-escalation trial. Lancet. 2014;384(9937):37-44. https://doi.org/10.1016/S0140-6736(13) 61841-3.

12. Khalil A, Würthwein G, Golitsch J, et al. Pre-existing antibodies against polyethylene glycol reduce asparaginase activities on first administration of pegylated $E$. coli asparaginase in children with acute lymphocytic leukemia. Haematologica. 2020. https://doi. org/10.3324/haematol.2020.258525.

13. van der Sluis IM, Vrooman LM, Pieters R, et al. Consensus expert recommendations for identification and management of asparaginase hypersensitivity and silent inactivation. Haematologica. 2016;101(3):279-85. https://doi.org/10.3324/haematol.2015. 137380.

14. Tong WH, Pieters R, Kaspers GJL, et al. A prospective study on drug monitoring of PEGasparaginase and Erwinia asparaginase and asparaginase antibodies in pediatric acute lymphoblastic leukemia. Blood. 2014. https://doi.org/10.1182/ blood-2013-10-534347.

15. Ko RH, Jones TL, Radvinsky D, et al. Allergic reactions and antiasparaginase antibodies in children with high-risk acute lymphoblastic leukemia: a children's oncology group report. Cancer. 2015;121(23):4205-11. https://doi.org/10.1002/cncr.29641.

16. Würthwein G, Lanvers-Kaminsky C, Siebel C, et al. Population pharmacokinetics of PEGylated asparaginase in children with acute lymphoblastic leukemia: treatment phase dependency and predictivity in case of missing data. Eur J Drug Metab Pharmacokinet. 2021;46(2):289-300. https://doi.org/10.1007/ s13318-021-00670-8.

17. Würthwein G, Lanvers-Kaminsky C, Hempel G, et al. Population pharmacokinetics to model the time-varying clearance of the PEGylated Asparaginase Oncaspar ${ }^{\circledR}$ in children with acute lymphoblastic leukemia. Eur J Drug Metab Pharmacokinet. 2017;42(6):955-63. https://doi.org/10.1007/s13318-017-0410-5.

18. Wählby U, Thomson AH, Milligan PA, Karlsson MO. Models for time-varying covariates in population pharmacokinetic-pharmacodynamic analysis. Br J Clin Pharmacol. 2004;58(4):367-77. https://doi.org/10.1111/j.1365-2125.2004.02170.x.

19. Icon Development Solutions. NONMEM users's guides. Ellicott City, MD: Icon Development Solutions; 2009. 
20. R Core Team. R: a language and environment for statistical computing. Vienna: R Foundation for Statistical Computing; 2021.

21. RStudio Team. RStudio: integrated development environment for R. Boston, MA, USA: RStudio, Inc.; 2021.

22. Lindbom L, Pihlgren P, Jonsson EN, Jonsson N. PsN-Toolkita collection of computer intensive statistical methods for nonlinear mixed effect modeling using NONMEM. Comput Methods Programs Biomed. 2005;79(3):241-57. https://doi.org/10.1016/j. cmpb.2005.04.005.

23. Keizer RJ, Karlsson MO, Hooker A. Modeling and simulation workbench for NONMEM: tutorial on Pirana, PsN, and Xpose. CPT Pharmacometrics Syst Pharmacol. 2013;2:e50. https://doi. org/10.1038/psp.2013.24.

24. Silverman LB, Gelber RD, Dalton VK, et al. Improved outcome for children with acute lymphoblastic leukemia: results of DanaFarber Consortium Protocol 91-01. Blood. 2001;97(5):1211-8. https://doi.org/10.1182/blood.v97.5.1211.

25. Abshire TC, Pollock BH, Billett AL, Bradley P, Buchanan GR. Weekly polyethylene glycol conjugated L-asparaginase compared with biweekly dosing produces superior induction remission rates in childhood relapsed acute lymphoblastic leukemia: a Pediatric Oncology Group study. Blood. 2000;96(5):1709-15. https://doi. org/10.1182/blood.V96.5.1709.

26. Gupta S, Wang C, Raetz EA, et al. Impact of asparaginase discontinuation on outcome in childhood acute lymphoblastic leukemia: a report from the Children's Oncology Group. J Clin Oncol. 2020;38(17):1897-905. https://doi.org/10.1200/JCO.19.03024.
27. Gottschalk Højfeldt S, Grell K, Abrahamsson J, et al. Relapse risk following truncation of pegylated asparaginase in childhood acute lymphoblastic leukemia. Blood. 2021;137(17):2373-82. https:// doi.org/10.1182/blood.2020006583.

28. Kloos RQH, Uyl-de Groot CA, van Litsenburg RRL, Kaspers GJL, Pieters R, van der Sluis IM. A cost analysis of individualized asparaginase treatment in pediatric acute lymphoblastic leukemia. Pediatr Blood Cancer. 2017. https://doi.org/10.1002/pbc.26651.

29. Kloos RQH, Mathôt R, Pieters R, van der Sluis IM. Individualized dosing guidelines for PEGasparaginase and factors influencing the clearance: a population pharmacokinetic model. Haematologica. 2020. https://doi.org/10.3324/haematol.2019.242289.

30. Poppenborg SM, Wittmann J, Walther W, et al. Impact of antiPEG IgM antibodies on the pharmacokinetics of pegylated asparaginase preparations in mice. Eur J Pharm Sci. 2016;91:122-30. https://doi.org/10.1016/j.ejps.2016.06.007.

31. Edlund H, Steenholdt C, Ainsworth MA, et al. Magnitude of increased infliximab clearance imposed by anti-infliximab antibodies in Crohn's disease is determined by their concentration. AAPS J. 2017;19(1):223-33. https://doi.org/10.1208/ s12248-016-9989-8.

32. Mondelaers V, Ferster A, Uyttebroeck A, et al. Prospective, real-time monitoring of pegylated Escherichia coli and Erwinia asparaginase therapy in childhood acute lymphoblastic leukaemia and non-Hodgkin lymphoma in Belgium. Br J Haematol. 2020;190(1):105-14. https://doi.org/10.1111/bjh.16495.

\section{Authors and Affiliations}

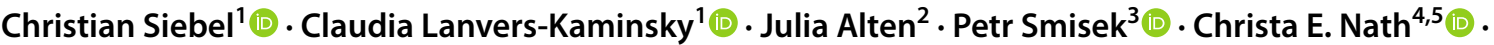

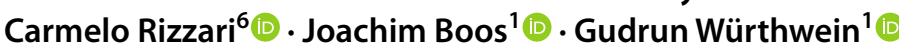

1 Department of Paediatric Haematology and Oncology, University Children's Hospital Muenster,

Albert-Schweitzer-Campus 1, A1, 48149 Muenster, Germany

2 Department of Paediatrics, Christian-Albrechts-University Kiel and University Medical Centre Schleswig-Holstein, Kiel, Germany

3 Department of Paediatric Haematology and Oncology, Charles University and University Hospital, Motol, Prague, Czech Republic
4 Biochemistry Department and Cancer Centre for Children, The Children's Hospital at Westmead, Westmead, NSW, Australia

5 Faculty of Health and Medicine, University of Sydney, Sydney, NSW, Australia

6 Paediatric Haematology-Oncology Unit, Department of Paediatrics, University of Milano-Bicocca, MBBM Foundation, ASST-Monza, Monza, Italy 